\title{
Intrinsic Lévy behaviour in organisms - searching for a mechanism
}

\section{Comment on "Liberating Lévy walk research from the shackles of optimal foraging" by}

\section{A.M. Reynolds}

\author{
David W. Sims
}

Marine Biological Association of the United Kingdom, The Laboratory, Citadel Hill, Plymouth PL1 2PB, UK

Ocean and Earth Science, National Oceanography Centre Southampton, University of Southampton, Waterfront Campus, European Way, Southampton SO14 3ZH, UK

Centre for Biological Sciences, Building 85, University of Southampton, Highfield Campus, Southampton SO17 1BJ, UK

Email:dws@mba.ac.uk

Keywords: Lévy walks; Lévy flights; anomalous diffusion; movement ecology; neurobiology; foraging; scale-free; power laws

The seminal papers by Viswanathan and colleagues in the late 1990s [1,2] proposed not only that scale-free, superdiffusive Lévy walks can describe the free-ranging movement patterns observed in animals such as the albatross [1], but that the Lévy walk was optimal for searching for sparsely and randomly distributed resource targets [2]. This distinct advantage, now shown to be present over a much broader set of conditions than originally theorised [3], implied that the Lévy walk is a search strategy that should be found very widely in organisms [4]. In the years since there have been several influential empirical studies showing that Lévy walks can indeed be detected in the movement patterns of a very broad range of taxa, from jellyfish, insects, fish, reptiles, seabirds, humans [5-10], and even in the fossilised trails of extinct invertebrates [11]. The broad optimality and apparent deep evolutionary origin of movement (search) patterns that are well approximated by Lévy walks led to the development of the Lévy flight foraging (LFF) hypothesis [12], which states that "since Lévy flights and walks can optimize search efficiencies, therefore natural selection should have led to adaptations for Lévy flight foraging".

The idea that organism search strategies naturally evolved to exploit optimal Lévy patterns has gathered pace in recent years $[5,7,9,11]$. To account for observed Lévy-like behaviour - by which I mean behaviour patterns well approximated by a truncated Lévy distribution - it has been hypothesized that (i) scale-free activities may arise from intrinsic processes [9,11,13-16], (ii) that behavioural adaptations to changes in environmental resources may cue the switching between localized Brownian and Lévy random searching [5,7], or (iii) that sensory interactions with heterogeneous environments may give rise to Lévy movement patterns (an emergent phenomena) $[17,18]$. However, the origins of such potential mechanisms remain elusive. 
The review by Reynolds [19] is a timely synthesis of this burgeoning topic. The review proposes that the Lévy flight foraging hypothesis may be too narrowly focused on optimal foraging to be ideal for framing questions aimed at exploring how scale-free movements and behaviours may arise. I do not entirely agree with this position. I hold the view that to understand the mechanisms underlying the observed scale-free (Lévy) patterns it will be necessary to consider both intrinsic and extrinsic processes, in addition to behavioural adaptations that are flexible and including cognitive processes such as learning and sociality. In my opinion a new hypothesis would be particularly valuable if it could unify these different aspects. Whilst I appreciate that the LFF hypothesis may not achieve this aim for all behaviour patterns observed, it is evident that the free hypothesis outlined [19] does not succeed entirely in this endeavour either.

The LFF hypothesis essentially considers natural selection as a driver for widely observed Lévy search patterns of organisms. In this idea it is the competition occurring between individuals that favours the survival of those approaching or exhibiting optimal Lévy searches (for resources such as food or mates) [3]. Reynolds [19] proposes a new synthesis supporting a new hypothesis - the 'free Lévy flight hypothesis' - which states that "Lévy flights emerge spontaneously and naturally from innate behaviours and inocuous responses to the environment but, if advantageous, then there could be selection against losing them". The choice of the word 'innate' is problematic because it is not defined, even though historically it has been the source of much controversy in the behavioural sciences [20]. The normal use of the term 'innate' has been to describe a behaviour which was adapted to its present function by natural selection. A so-called innate (inborn) behaviour can be a complex behaviour, one that is developmentally fixed, but this does not necessarily mean it is independent of environmental influences or genetically fixed. Indeed, there are very few behaviour patterns that run to completion regardless of information from the environment and in the light of feedback from the results of an organism's own actions [20]. Nevertheless, it appears that 'innate behaviours' in the proposed free hypothesis is meant to signify behaviour that develops without example or practice and is fixed. Regardless of that contention, the principal idea of the free Lévy flight hypothesis is that Lévy patterns arise as a consequence of factors unrelated to behaviour (e.g. internal factors linked to physiology) [15] - with the assumption that Lévy characteristics are neutral - or that organisms interact with their heterogeneous environment such that Lévy patterns emerge [17]. It is proposed that if either of these are advantageous then natural selection will favour against the loss of these characteristics. On the face of it, the free Lévy flight hypothesis does not appear, as argued, to be a broader hypothesis than the LFF hypothesis. Rather, I would argue, they are somewhat different faces of the same coin.

The proposed free Lévy flight hypothesis is largely focused on explanations that favour the prevalence of scale-free behaviour as an intrinsic, spontaneous behaviour. However, this explanation is not outwith the LFF hypothesis in my view. In addition to it being possible for scalefree activities to be generated intrinsically $[13,15,16]$, it is also possible that an innate, intrinsic pattern is flexible, is under selection and becomes 'optimal' in the general environment in which a species finds itself. For example, the nematode worm (Caenorhabditis elegans) undertakes searching movements for food that show behavioural transition between an environmentally informed 'extrinsic' strategy that is influenced by recent experience and controls area-restricted searching, and a time-dependent 'intrinsic' strategy that reduces spatial oversampling and improves random encounter success [21]. A study using quantitative genetic analysis to examine the mechanisms underlying the behavioural transition decision of $C$. elegans to leave a food patch showed genetic 
variation and environmental cues converge on common neural circuits to regulate this behaviour [22]. This result suggests that behaviours linked to decisions to make longer movement steps away from depleted food patches ( $c f$. long steps in a Lévy walk) are heritable traits modifiable by the environment and not solely owing to a fixed and neutral intrinsic behaviour program or to emergent interactions with food patches. This emphasises the need to consider apparently 'innate' behaviours as flexible, i.e. they are capable of responding rapidly to environmental changes and are modified slowly by changes in the genome [22]. This modern understanding of 'innate', intrinsic behaviours sits well within the LFF hypothesis.

The free Lévy flight hypothesis also attempts to incorporate Lévy walks as an emergent property of environmental interaction. However, the arguments are not particularly convincing. For example, it is claimed that emergent Lévy walks are present in marine predators. With reference to previous observations of vertical Lévy movements in diving marine predators [5,7] Reynolds' uses his own analytical and simulation study of ocean turbulence to conclude that the numerical results were consistent with marine predators (mainly large fish species) changing their direction of travel upon encountering patches of relatively strong turbulence, the distribution of which was scale-free, but otherwise moving in straight lines; thus emerges the Lévy walk. However, this interpretation of vertebrate behaviour is entirely speculative; there is no empirical evidence that vertical displacements of oceanic fish respond to turbulence in this way, nevertheless no uncertainties surrounding this assumption were expressed in the review. Detailed evidence was also ignored about the foraging patterns of albatrosses to support the emergent Lévy walk idea by saying "it is now known that fish odours facilitate prey location in these birds". In contrast, the study referenced in the review, Nevitt et al. [23], actually indicates that only $46.8 \%$ of prey locations made by tracked albatrosses were achieved using olfactory clues, the others were seemingly random encounters. In more recent research it has also been shown that $74 \%$ of tracked albatrosses exhibited Lévy movements during foraging trips [9,24], suggesting therefore that significant numbers of prey encounters associated with Lévy foraging patterns are not likely due to emergent Lévy walks arising solely from interaction with prey odours. This empirical evidence undermines the general applicability of the free hypothesis in important ways, whereas the LFF hypothesis predicts such transitions in behaviour, for example between Brownian walks where resource targets (e.g. prey) are abundant and Lévy walks when targets are sparsely distributed $[3,7,25]$.

In conclusion, the review [19] is timely in drawing together studies that demonstrate scale-free activities in many different organisms, from single cells to humans. The idea that such patterns of activity are intrinsic and spontaneous is not new, however a renewed focus on understanding how intrinsic Lévy behaviours are generated within organisms is a great challenge worth taking up. It is possible that there is not one and the same mechanism underlying spontaneous scale-free patterns that may arise in animals as different in behaviour and morphological complexity as are fruit flies and sharks, for example. And whether search and other behaviours are under selection in different species may also vary greatly. But experiments that can differentiate between a true intrinsic behaviour pattern that converges to a Lévy walk and one that is mediated by the environment or by behavioural or cognitive adaptations will be a great first step along the path. 
Acknowledgements. DWS was funded by the UK Natural Environment Research Council and a Marine Biological Association Senior Research Fellowship. N. Humphries is thanked for discussions and for providing comments.

\section{References}

[1] Viswanathan $G M$, et al. Lévy flight search patterns of wandering albatrosses. Nature 1996;381:413-415.

[2] Viswanathan GM, et al. Optimizing the success of random searches. Nature 1999;401:911-914.

[3] Humphries NE, Sims DW. Optimal foraging strategies: Lévy walks balance searching and patch exploitation under a very broad range of conditions. Journal of Theoretical Biology 2014;358:179193.

[4] Viswanathan GM, da Luz MGE, Raposo EP, Stanley HE. The Physics of Foraging: An Introduction to Random Searches and Biological Encounters 2011;Cambridge University Press, New York.

[5] Sims DW, et al. Scaling laws of marine predator search behaviour. Nature 2008;451:1098-1102.

[6] Reynolds AM, et al. Displaced honey bees perform optimal scale-free search flights. Ecology 2007;88:1955-1961.

[7] Humphries NE, et al. Environmental context explains Lévy and Brownian movement patterns of marine predators. Nature 2010;465:1066-1069.

[8] Hays GC, et al. High activity and Lévy searches: jellyfish can search the water column like fish. Proceedings of the Royal Society B 2012;279:465-473.

[9] Humphries NE, Weimerskirch H, Queiroz N, Southall EJ, Sims DW. Foraging success of biological Lévy flights recorded in situ. Proceedings of the National Academy of Sciences of the U.S.A. 2012;109:7169-7174.

[10] Raichlen DA, et al. Evidence of Lévy walk foraging patterns in human hunter-gatherers. Proceedings of the National Academy of Sciences of the U.S.A. 2014;111:728-733.

[11] Sims DW, et al. Hierarchical random walks in trace fossils and the origin of optimal search behaviour. Proceedings of the National Academy of Sciences of the U.S.A. 2014;111:11073-11078.

[12] Viswanathan GM, Raposo EP, da Luz MGE Lévy flights and superdiffusion in the context of biological encounters and random searches. Physics of Life Reviews 2008;5:133-150.

[13] Maye A, Hsieh C-H, Sugihara G, Brembs B. Order in spontaneous behaviour. PLoS ONE 2007;2:e443.

[14] Proekt A, Banavar JR, Maritan A, Pfaff DW. Scale invariance in the dynamics of spontaneous behaviour. Proceedings of the National Academy of Sciences of the U.S.A. 2012;109:10564-10569. 
[15] Wearmouth VJ, et al. Scaling laws in ambush predator 'waiting' behaviour are tuned to a common ecology. Proceedings of the Royal Society B 2014;281:20132997.

[16] Kölzsch A, et al. Experimental evidence for inherent Lévy search behaviour in foraging animals. Proceedings of the Royal Society B 2015;282:20150424.

[17] Boyer D, et al. Scale-free foraging by primates emerges from their interaction with a complex environment. Proceedings of the Royal Society B 2006;273:1743-1750.

[18] Miramontes O, Boyer D, Bartumeus F. The effects of spatially heterogeneous prey distributions on detection patterns in foraging seabirds. PLoS ONE 2012;7:e34317.

[19] Reynolds AM. Liberating Lévy walk research from the shackles of optimal foraging. Physics of Life Reviews 2015; this issue.

[20] Slater PJB. Essentials of Animal Behaviour 1999; Cambridge University Press, Cambridge.

[21] Salvador LCM, Bartumeus F, Levin SA, Ryu WS. Mechanistic analysis of search behaviour of Caenorhabditis elegans. Journal of the Royal Society Interface 2014;11:20131092.

[22] Bendesky A, Tsunozaki M, Rockman MV, Kruglyak L, Bargmann Cl. Catecholamine receptor polymorphisms affect decision-making in C. elegans. Nature 2011;472:313-318.

[23] Nevitt GA, Losekoot M, Weimerskirch H. Evidence for olfactory search in wandering albatross, Diomedea exulans. Proceedings of the National Academy of Sciences of the U.S.A. 2008;105:45764581.

[24] Humphries NE, Weimerskirch H, Sims DW. A new approach to objective identification of turns and steps in organism movement data relevant to random walk modelling. Methods in Ecology and Evolution 2013;4:930-938.

[25] Sims DW, Humphries NE, Russell WB, Bruce BD. Lévy flight and Brownian search patterns of a free-ranging predator reflect different prey field characteristics. Journal of Animal Ecology 2012;81:432-442. 